\title{
The Crucial Things in Science often Happen Quite Unexpectedly-Das Entscheidende in der Wissenschaft Geschieht oft Ganz Unerwartet (K. Alex Müiller)
}

\author{
Reinhard K. Kremer ${ }^{1, *(D)}$, Annette Bussmann-Holder ${ }^{1}$, Hugo Keller ${ }^{2}$ and Robin Haunschild ${ }^{1}(\mathbb{D}$ \\ 1 Max Planck Institute for Solid State Research, Heisenbergstrasse 1, 70569 Stuttgart, Germany; \\ a.bussmann-holder@web.de (A.B.-H.); R.Haunschild@fkf.mpg.de (R.H.) \\ 2 Physik Institut der Universität Zürich, Winterthurerstr. 190, 8057 Zürich, Switzerland; keller@physik.uzh.ch \\ * Correspondence: rekre@fkf.mpg.de; Tel.: +49-711-689-1688
}

Received: 10 June 2020; Accepted: 28 June 2020; Published: 1 July 2020

\begin{abstract}
We analyzed the publication output of one of the 1987 Nobel Prize awardees, K. Alex Müller, using bibliometric methods. The time-dependent number of publications and citations and the network with respect to the coauthors and their affiliations was studied. Specifically, the citation history of the Nobel Prize awarded 1986 article on "Possible high-temperature superconductivity in the Ba-La-Cu-O system" has been evaluated in terms of the overall number of articles on superconductivity and the corresponding citations of other most frequently referenced articles. Thereby, a publication with "delayed recognition" was identified.
\end{abstract}

\section{Introduction}

Rarely in the history of physics has a discovery had such a prompt and enduring impact as the observation of high-temperature superconductivity (high- $T_{\mathrm{c}}$ ) in the Ba-La-Cu-O system by J. Georg Bednorz and K. Alex Müller in 1986 [1]. The unexpected disclosure of superconductivity at temperatures about twice as high as had been known before [2,3] triggered immediate (sometimes hectic and often very competitive and controversial) research activity targeted at the synthesis of compounds with even higher $T_{\mathrm{c}}$ 's and the still contentious theoretical explanation of high- $T_{\mathrm{c}}$ superconductivity. The bursting reaction in the search for new and deeper insight into already known high- $T_{\mathrm{c}}$ materials is reflected in the instantaneous rise of the number of publications indexed by the Web of Science (WoS) $[4,5]$ before and after 1986 (see Figure 1).

Figure 1 shows that after 1986 about 30\% of the publications related to superconductivity covered high- $T_{\mathrm{c}}$ research (see [6-8]). Since 1986, the number of publications dealing with superconductivity essentially remains constant at a high level of about 6500 per year, whereas the number of high- $T_{\mathrm{c}}$ papers decreased gradually after a peak in 1991 with approximately 3100 publications and currently levels off at about 1400 publications per year. A closer inspection of Figure 1 also reveals a couple of historically very interesting aspects: After the discovery of superconductivity in mercury by H. Kamerling Onnes in 1911 [9] there was an extended period lasting until the late 1950s with a continuous but moderate increase of the publication output with about 10 to 50 superconductivity publications per year. Note the plunge in the number of publications in the late years of the Second World War. Certainly triggered by the development of the Bardeen-Cooper-Schrieffer (BCS) theory of superconductivity [10] but very likely also by the first placing of a satellite into Earth's orbit by the Soviet Union in October 1957, the number of publications rose steeply in the late 1950s and early 1960s [11]. This event, perceived in the Western world as the 'Sputnik crisis', initiated massive funding increase into science, especially in the USA [12]. As a consequence, the number of scientists working in the field and the publication 
output increased steeply after 1957 [13,14]. The data also show that after 1970 the publication output stalled and flattened, because no new discoveries were made, and increased dramatically after 1987.

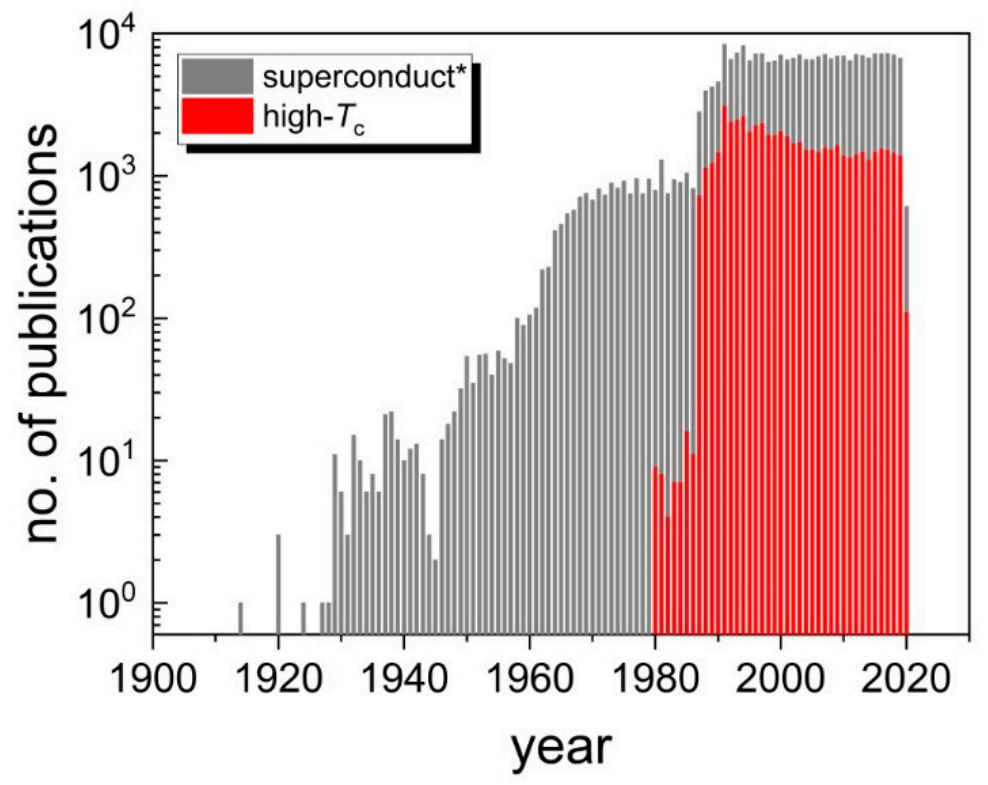

Figure 1. Number of publications indexed by the Web of Science (WoS) either with the keywords 'superconduct*' (grey) or 'high- $\mathrm{T}_{\mathrm{C}}$ ' (red). [4].

\section{The Publication Output of Original Research Publications}

K. Alex Müller began his scientific career with his $\mathrm{PhD}$ thesis entitled 'Paramagnetische Resonanz von $\mathrm{Fe}^{3+}$ in $\mathrm{SrTiO}_{3}$ Einkristallen' submitted to the ETH Zürich in 1958. In his thesis he summarized his microwave resonance experiments on spin $S=5 / 2$ ions substituted for $\mathrm{Ti}$ in $\mathrm{SrTiO}_{3}$, a compound which fascinated him during all his future scientific activity. The thesis was published in the Helvetica Physica Acta in 1958 [15]. The results of his thesis were summarized in a short single-authored Physical Review Letter (PRL) published in 1959, already mentioning his new affiliation at the Battelle Memorial Institute in Geneva [16]. This PRL referenced a publication, apparently K. Alex Müller's very first one (possibly published more or less simultaneously with his $\mathrm{PhD}$ thesis), in the proceedings of the 7th Colloquium Ampère held in 1958 in Geneva (Résonance paramagnétique du $\mathrm{Cr}^{3+}$ dans des monocristaux de $\mathrm{SrTiO}_{3}$ ) [17].

Figure 2a shows the number of papers published by K. Alex Müller per year indexed by the WoS as source items beginning from his first Physical Review Letter, which appeared in 1959. Figure $2 b$ is a 'vintage' diagram, showing the number of citations for papers published in the particular year.

Unfortunately, the 'Archives des Sciences éditées par la Société de Physique et d'Histoire Naturelle de Genève' are not indexed by the WoS and a reliable bibliometric analysis of this publication cannot be carried out [18].

In total, K. Alex Müller published 311 papers in the 61 years of his scientific activity until 2019. Until 1986, he authored between three and eight publications per year (see Figure 2a). Interestingly, this number did not increase significantly over the following years, except in the first two immediate years after the seminal paper on the discovery of high- $T_{\mathrm{c}}$ superconductivity in 1986 [1] when he authored or co-authored up to 30 papers in 1987 and 22 in 1988 (i.e., about one-sixth of Müller's publications appeared in these two years). It is worth mentioning that the number of publications dealing with $\mathrm{SrTiO}_{3}$ (50) is almost as high as those covering cuprates (52). Including 11 additional papers which treated titanates in general $\left(\right.$ mostly $\left.\mathrm{BaTiO}_{3}\right)$, the number of publications on titanates (61) even exceeds that on cuprates. 
Figure $2 \mathrm{~b}$ displays a 'vintage' diagram showing the number of citations to papers published in a particular year. Besides the citation spikes following immediately after the discovery of high- $T_{\mathrm{C}}$ superconductivity, two more years stand out, namely the years 1979 and 1996. The high citation number in 1979 is due to one particular paper, which also exhibited an unusual citation history (see details below). In 1996, the high citation number is owed to two publications on $\mathrm{La}_{1-\mathrm{x}} \mathrm{Ca}_{\mathrm{x}} \mathrm{MnO}_{3+\mathrm{y}}$, namely an article in Nature together with Zhao, Conder, and Keller on giant oxygen isotope shifts (622 citations) and a PRL published jointly with Shengelaya, Zhao, and Keller providing electron paramagnetic resonance (EPR) evidence for Jahn-Teller polaron formation (215 citations) (see Table 2 and Ref. [19] for the detailed references).

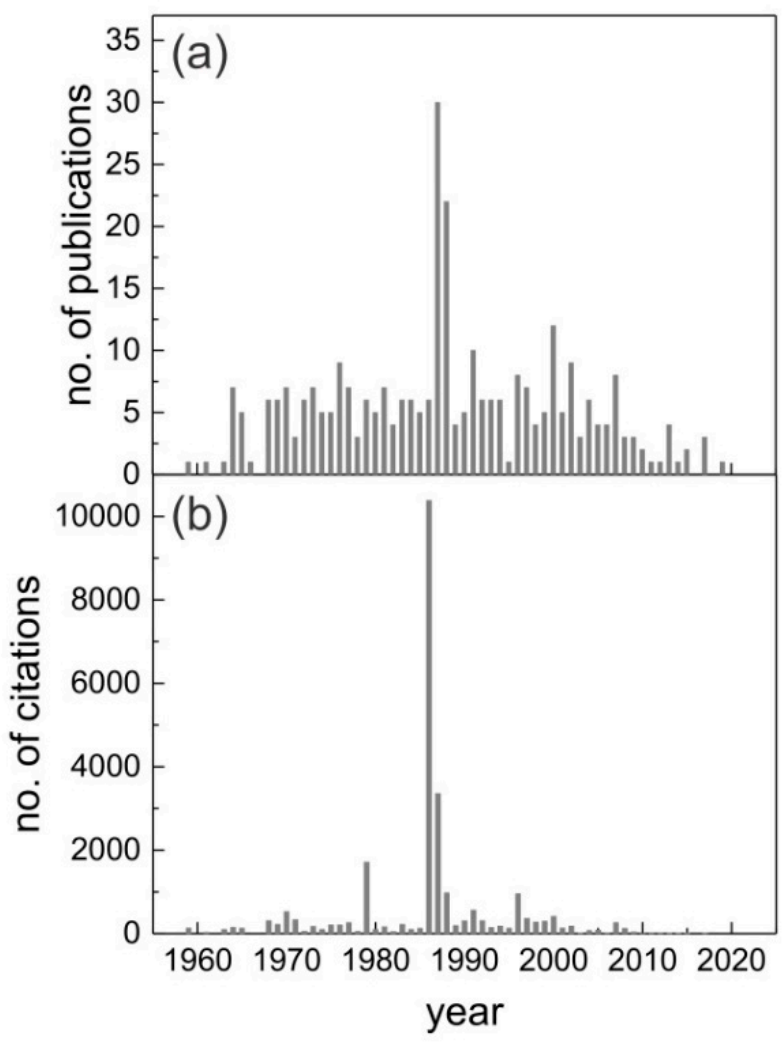

Figure 2. (a) Number of papers published by K. Alex Müller per year indexed by the WoS as source items beginning from his first Physical Review Letter, which appeared in 1959. (b) 'Vintage' diagram showing the number of citations for papers published in the particular year.

K. Alex Müller's publication output compares well, for example, with that of V. L. Ginzburg, another Nobel prize winner for "Pioneering Contributions to the Theory of Superconductors and Superfluids", whose scientific activity covered a similar period of time as of K. Alex Müller's [20].

\section{Editorial Activities}

Over his long-lasting scientific activity, K. Alex Müller edited and co-edited numerous collections of lectures, conference proceedings, and series volumes, to which he usually contributed an article or a chapter. Table 1 provides a compilation of Müller's editorial activity. Clearly, in the 1970s and 1980s these books focused around phase transition-related phenomena. Later, after the discovery of high- $T_{\mathrm{c}}$ superconductivity, these were in his focus. Noteworthy are the two conference proceedings of international symposia on phase separation problems in cuprate superconductors held in Erice and Cottbus in 1992 and 1993, respectively. These meetings and the contributions summarized in the proceedings reflect Müller's early interest in essential inhomogeneity in high- $T_{\mathrm{C}}$ superconductors. 
Table 1. Compilation of K. Alex Müller's editorial activities.

Müller, K.A.; Rigamonti, A. Local Properties at Phase Transitions. Proprietà Locali alle Transizioni di Fase, Societa Italiana di Fisica Bologna, 1976.

Müller, K.A.; Thomas, H. Structural Phase Transitions I. Springer-Verlag, Berlin, Heidelberg, New York, 1981.

Müller, K.A.; Thomas, H. Structural Phase Transitions II. Springer-Verlag, Berlin, Heidelberg, New York, 1991.

Bednorz, J.G.; Müller, K.A. Earlier and Recent Aspects of Superconductivity, Lectures from the International School, Erice, Trapani, Sicily, 4-16 July, 1989. Springer Verlag, 1st Edition 1990, 2nd Edition 1991.

Müller, K.A.; Benedek, G. Proceedings of the Workshop on Phase Separation in Cuprate Superconductors. Erice, Italy, World Scientific Publishing, 1993.

Sigmund, E.; Müller, K.A. Phase Separation in Cuprate Superconductors. Proceedings of the second international workshop on 'Phase Separation in Cuprate Superconductors', 4-10 September, 1993. Cottbus, Germany, Springer-Verlag, 1994.

Müller, K.A.; Bussmann-Holder, A. Superconductivity in Complex Systems, Structure and Bonding 114, Springer-Verlag, 2005.

Müller, K.A.; Kool, T.W. Properties of Perovskites and Other Oxides. World Scientific, 2010.

Müller, K.A. Letters Section in the Journal of Superconductivity and Novel Magnetism, published by Springer Nature.

\section{The Citation Record-Overall}

In his long scientific career, K. Alex Müller's publications were cited about 25,000 times. By far the highest citation count of all of his publications was acquired by the Zeitschrift für Physik article of 1986 on 'Possible High- $T_{\mathrm{c}}$ Superconductivity in the BA-LA-CU-O System' (see Figure 3). Until February 2020 , it was cited more than an exceptional 10,000 times. Note that we did not find any publication on superconductivity that was cited more frequently. The citation count for this publication peaks two years after its appearance at a value of about 1250 citations per year. Subsequently, the citations decrease to pass through a broad minimum around the year 2005, whereupon they start to grow again until today. Such a second increase is remarkable and must be attributed to the discovery of new classes of high- $T_{\mathrm{c}}$ superconductors, e.g., $\mathrm{MgB}_{2}$ or the iron-based superconductors. Forty of K. Alex Müller's 311 scientific papers acquired more than 100 citations. The 10 most frequently cited papers are compiled in Table 2.

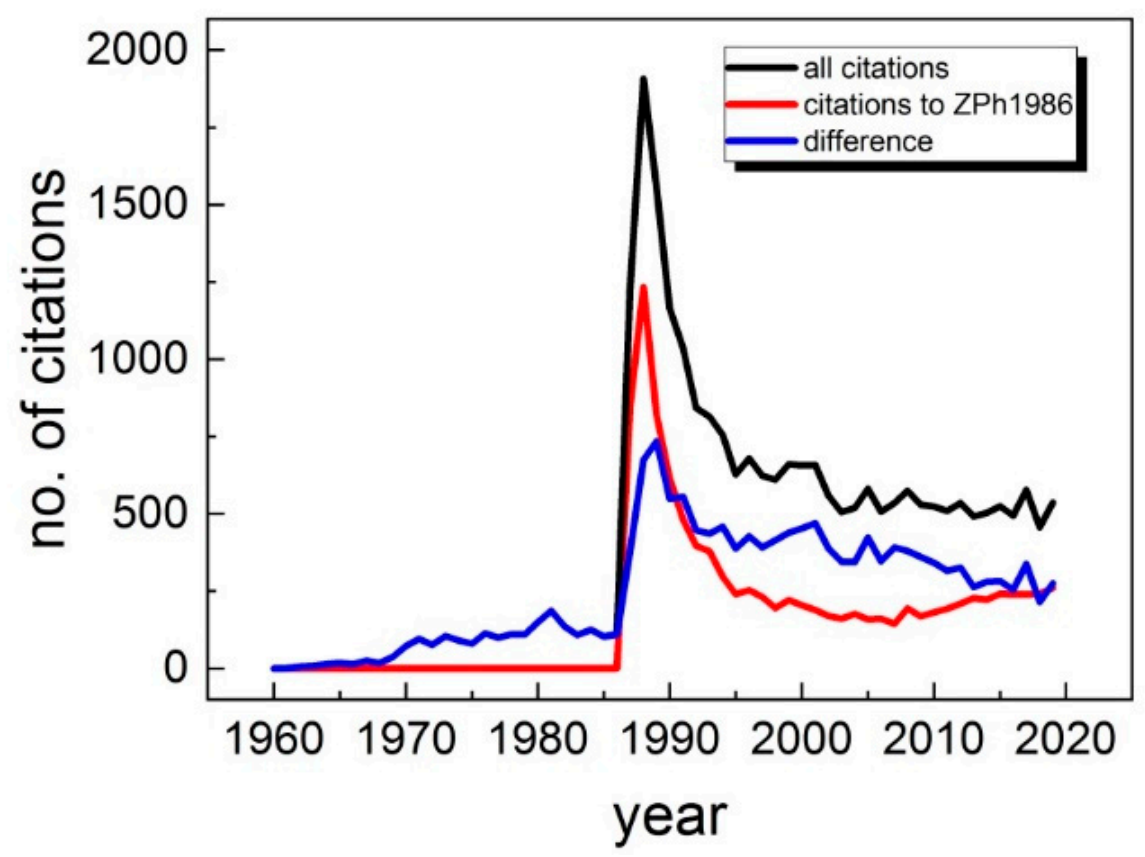

Figure 3. Time evolution of the number of citations to K. Alex Müller's papers. The black solid line gives the number of all citations per year, the red solid line the citations per year of the article in Zeitschrift für Physik in 1986 [1], and the blue solid line the difference between the first and the latter. 
Table 2. The 10 most cited publications authored and co-authored by K. Alex Müller (search date February 2020), ordered by their citation count.

1. Bednorz, J.G.; Müller, K.A.

Possible High-Tc Superconductivity in the BA-LA-CU-O System. Z. Phys. B Condens. Matter 1986, 64, 189-193. Doi: $10.1007 / \mathrm{BF} 01303701$.

times cited: 10210

2. Müller, K.A.; Burkard, H.

$\mathrm{SrTiO}_{3}$-Intrinsic Quantum Para-Electric Below 4-K. Phys. Rev. B 1979, 19, 3593-3602.

Doi: 10.1103/PhysRevB.19.3593.

times cited: 1196

3. Müller, K.A.; Takashige, M.; Bednorz, J.G.

Flux Trapping and Superconductive Glass State in $\mathrm{La}_{2} \mathrm{CuO}_{4-y}$ :Ba. Phys. Rev. Lett. 1987, 58, 1143-1146.

Doi: 10.1103/PhysRevLett.58.1143.

times cited: 1094

4. Zhao, G.M.; Conder, K.; Keller, H.; Müller, K.A.

Giant Oxygen Isotope Shift in the Magnetoresistive Perovskite $\mathrm{La}_{1-\mathrm{x}} \mathrm{CaxMnO}_{3+\mathrm{y}}$. Nature 1996, 381, 676-678.

Doi: $10.1038 / 381676 a 0$.

times cited: 622

5. Deutscher, G.; Müller, K.A.

Origin of Superconductive Glassy State and Extrinsic Critical Currents in High-Tc Oxides. Phys. Rev. Lett. 1987, 59, 1745-1747. Doi: 10.1103/PhysRevLett.59.1745.

times cited: 584

6. Blazey, K.W.; Müller, K.A.; Bednorz, J.G.; Berlinger, W.; Amoretti, G.; Buluggiu, E.; Vera, A.; Matacotta, F.C. Low-Field Microwave-Absorption in the Superconducting Copper Oxides. Phys. Rev. B 1987, 36, 7241-7243. DOI: 10.1103/PhysRevB.36.7241.

times cited: 327

7. Bednorz, J.G.; Müller, K.A.

Perovskite-Type Oxides - The New Approach to High-Tc Superconductivity. Rev. Modern Phys. 1988, 60, 585-600. Doi: 10.1103/RevModPhys.60.585.

times cited: 306

8. Zhao, G.M.; Hunt, M.B.; Keller, H.; Müller, K.A.

Evidence for Polaronic Supercarriers in the Copper Oxide Superconductors $\mathrm{La}_{2-x} \mathrm{Sr}_{x} \mathrm{CuO}_{4}$. Nature 1997, 385, 236-239. Doi: 10.1038/385236a0.

times cited: 273

9. Müller, K.A.; Berlinger, W.; Waldner, F.

Characteristic Structural Phase Transition in Perovskite-Type Compounds. Phys. Rev. Lett. 1968, 21, 814-817.

Doi: 10.1103/PhysRevLett.21.814.

times cited: 267

10. Šimanek, E.; Müller, K.A.

Covalency and Hyperfine Structure Constant-A of Iron Group Impurities in Crystals. J. Phys. Chem. Solids 1970, 31, 1027-1040. Doi: 10.1016/0022-3697(70)90313-6.

times cited: 266

\section{Citation History of Selected Highly Cited Publications}

The citation record as a function of time of a particular article, colloquially called its 'citation history', is specific for every article. However, it often follows a similar pattern [20]. Typically, it takes about one or two years until the citation count markedly starts to rise. Often, the citation count reaches a peak after about three years with a subsequent decrease when newer research results gradually become available. Such exemplary pattern can be observed for three of the four next highest cited publications of Müller, namely those in collaboration with Müller, Takashige, Bednorz, PRL 1987, Zhao, Conder, Keller, Müller, Nature 1996, and Deutscher and Müller, PRL 1987 (see Figure 4). However, the paper by Müller and Burkhard, Physical Review B (PRB) 1979 [21] (about 1200 total citations) entitled ' $\mathrm{SrTiO}_{3}$-Intrinsic quantum para-electric below $4 \mathrm{~K}$ ' exhibits a remarkably different citation history. Published in 1979, it was barely referenced until about 1990. Afterwards, it picked up and kept on increasing until now. It appears that the research reported in this article was well ahead of its time. Publications showing such delayed recognition have sometimes been labeled as 'sleeping beauties'. 


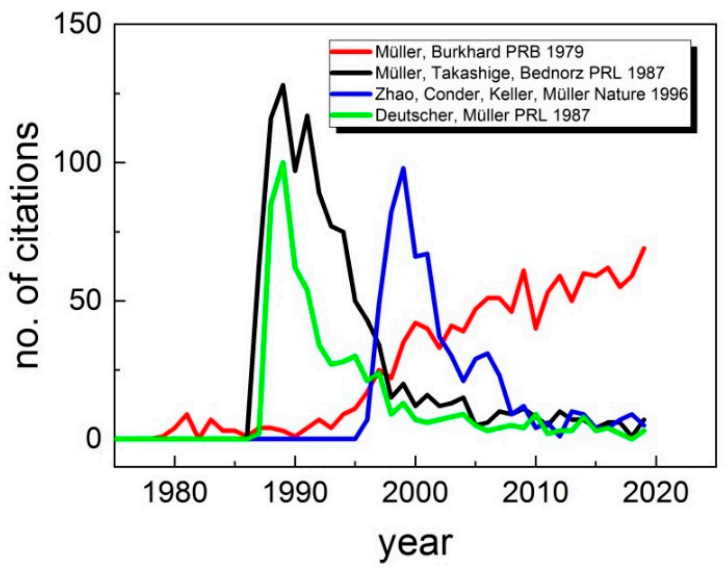

Figure 4. Citation history of the next four highest cited articles of K. Alex Müller besides the 1986 Zeitschrift für Physik paper. Note the unusual citation history characteristic of delayed recognition of the Müller and Burkhard article on quantum para-electric behavior of $\mathrm{SrTiO}_{3}$. For the detailed references see Table 2 .

\section{The Co-Author Network-People}

Figure 5 displays an overview (generated using the software VOSviewer [22]) of the co-authors with whom Müller published more than five papers. Above all, ranks J. Georg Bednorz from IBM Rüschlikon (58), with whom he shared the 1987 Nobel Prize. K. Alex Müller continuously emphasized the amount of competent work and inspiration he received from the constant and fruitful assistance of Walter Berlinger. Even nowadays, it is by no means customary to include technical staff as a co-author in a scientific paper. However, K. Alex Müller never ceased to acknowledge Berlinger's work and, consequently, the number of joint publications with Berlinger (55) ranks almost as high as that with Bednorz. K.W. Blazey, also from IBM Rüschlikon, is another regularly named co-author. Other frequently appearing co-authors are H. Keller and his postdoctoral fellow A. Shengelaya and their jointly supervised PhD student G. M. Zhao, all affiliated with the University in Zürich. E. Kaldis, K. Conder, and J. Karpinski from the ETH Zürich were frequent and highly appreciated sources of exquisite samples and crystals. With colleagues from Germany and particularly the Max-Planck-Institute (MPI) for Solid State Research in Stuttgart, K. Alex Müller had a long-lasting collaboration, resulting in several joint publications with A. Bussmann-Holder and A. Simon.

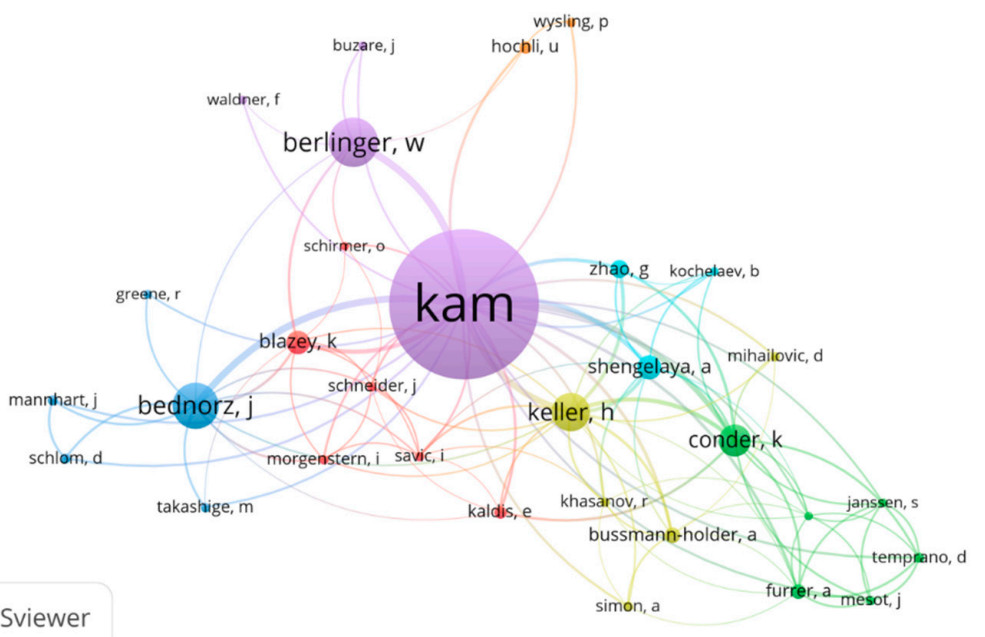

Figure 5. K. Alex Müller's main co-authors. The diameters of the circles correspond to the number of joint publications. Only co-authorships with at least five joint papers are included. 


\section{Countries}

Not surprisingly, K. Alex Müller's main co-authors were affiliated with research institutions in his home country Switzerland (see Figure 6, also generated using the software VOSviewer [22]), which are by far dominating the co-authorship network. Outside Switzerland, the co-author network comprised colleagues from 16 countries (one disconnected country, The Netherlands, was removed). The immediate neighbors, France and Germany (note that Germany includes Western Germany before the reunification), rank second followed by the USA. However, there are also substantial numbers of co-authors from Russia (note that Russia includes the Soviet Union) and smaller countries like Georgia, Slovenia, and Estonia

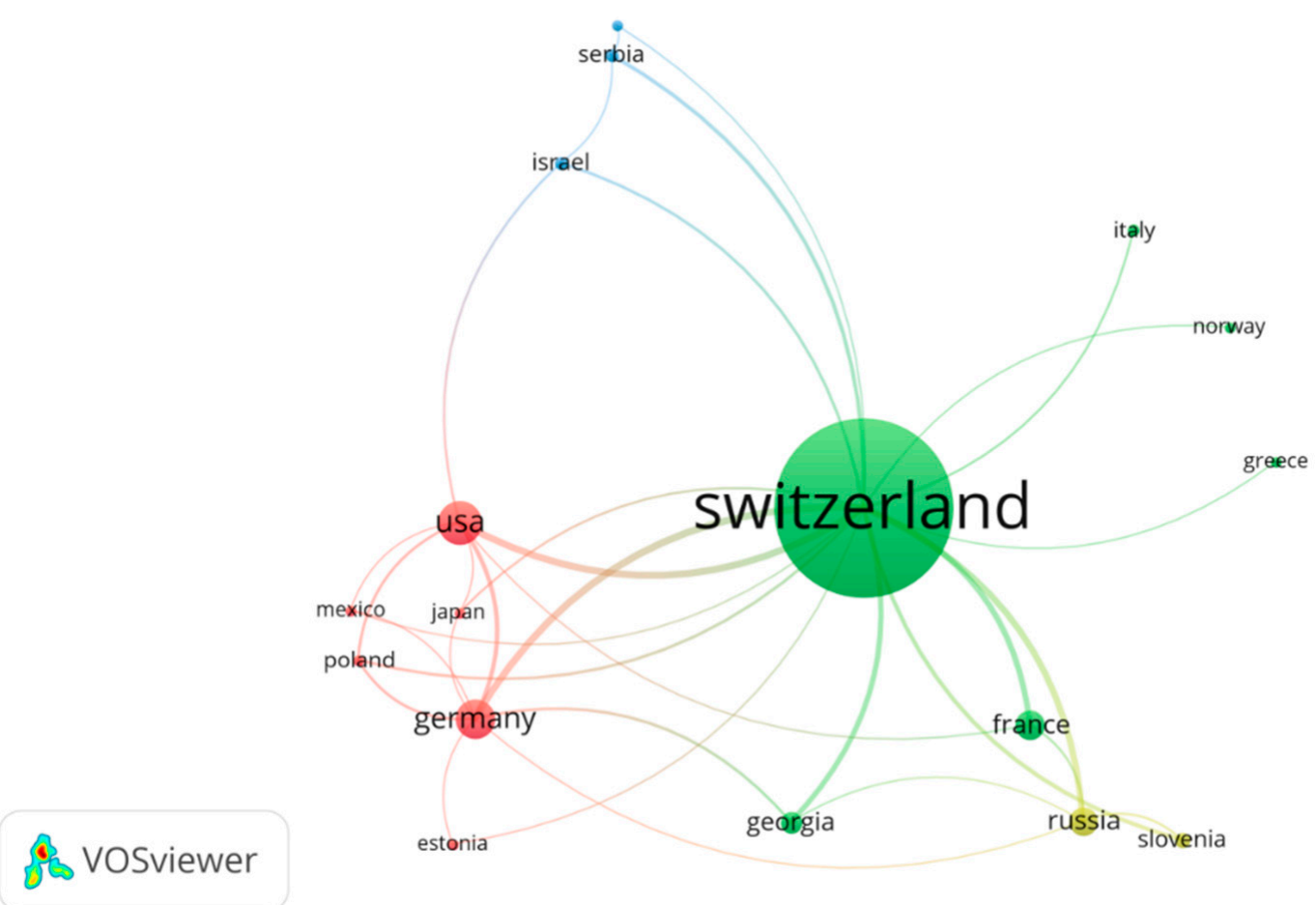

Figure 6. Country of affiliation of K. Alex Müller's main co-authors with whom he published at least one paper. The diameters of the circles correspond to the joint publication record with the named individual country.

\section{Conclusions}

The publication record and the citation history of K. Alex Müller's publications are exceptional and, from the bibliometric point of view, very interesting: Before J. Georg Bednorz and K. Alex Müller published their seminal paper on possible high- $T_{\mathrm{c}}$ superconductivity in Zeitschrift für Physik in 1986, Müller's scientific work focused on phase transition-related topics with an emphasis on perovskite materials, especially $\mathrm{SrTiO}_{3}$. Naturally, this changed after 1986 and the citation history of all his publications, subtracting out the high- $T_{\mathrm{c}}$ papers, increased by about a factor of four. Since its appearance, the Zeitschrift für Physik article itself has been cited more than 10,000 times. No other article on superconductivity has been cited more frequently. Forty of the 311 articles published by K. Alex Müller received more than 100 citations each. Müller's h-index amounts to 64 . Whereas most of his highest cited articles exhibit a citation history with a sharp peak shortly after publication and a steady decay thereafter (typical behavior), one noteworthy publication on $\mathrm{SrTiO}_{3}$, together with $\mathrm{H}$. Burkhard, displays an unusual, delayed recognition-type citation history. Currently, this publication continuous to attract attention and reached approximately 70 citations in 2019.

Funding: This research received no external funding. 
Acknowledgments: Very valuable discussions with W. Marx and Th. Scheidsteger as well as a careful reading of manuscript by S. Kremer are gratefully acknowledged.

Conflicts of Interest: The authors declare no conflict of interest.

\section{References and Notes}

1. Bednorz, J.G.; Müller, K.A. Possible high Tc superconductivity in the Ba-La-Cu-O system. Phys. Z. Condens. Matter 1986, 64, 189-193. [CrossRef]

2. Arrhenius, G.; Corenzwit, E.; Fitzgerald, R.; Hull, G.W.J.; Luo, H.L.; Matthias, B.T.; Zachariasen, W.H. Superconductivity of NB(3)(AL, GE) above 20.5 degrees K. Proc. Natl. Acad. Sci. USA 1968, 61, 621-628. [CrossRef] [PubMed]

3. One of the authors (RKK) recollects a seminar given by B. T. Matthias at the TU Darmstadt in the Elschner/Steglich seminar in the very early eighties of the last century. At the end of his lecture Matthias was asked what he estimates could be the maximum superconducting $T_{c}$. Matthias responded with a smile: This question apparently did not come as a great surprise and he must certainly have heard it many times before-and answered that he thinks that $T_{\mathrm{c}}$ will somehow level off between 30 and $35 \mathrm{~K}$.

4. The WoS as Provided by Clarivate Analytics was Used. Available online: https://apps.webofknowledge.com (accessed on 25 February 2020).

5. Birkle, C.; Pendlebury, D.A.; Schnell, J.; Adams, J. Web of Science as a data source for research on scientific and scholarly activity. Quant. Sci. Stud. 2020, 1, 363-376. [CrossRef]

6. At this occasion we want to point out that such bibliometric searches always are afflicted with some degree of uncertainty since they stick to the search terms. As an example, a publication containing the search term 'high- $T_{\mathrm{c}}$ ' but not 'superconduct $*$ ' shows up in the high- $T_{\mathrm{c}}$ category only, whereas if it contains both search terms it appears in both categories. How intriguing bibliometric searches can be, may be realized from the fact that the term 'high-temperature superconductors' has already been used for some A15 compounds in 1953/1954 (see e.g., Ref. [7]). Bibliometric searches for authors and citations are comparatively easier and generally more precise. For a detailed treatment of bibliometric analysis and its aspects see e.g., Ref. [8].

7. Gross, R.; Marx, A. Festkörperphysik; De Gruyter Mouton: Berlin, Germnay, 2012; p. 754.

8. Marx, W.; Bornmann, L. How to evaluate individual researchers working in the natural and life sciences meaningfully? A proposal of methods based on percentiles of citations. Scientometrics 2014, 89, 487-509.

9. Kamerling-Onnes, H. Further Experiments with Liquid Helium. C. On the Change of the Electric Resistance of Pure Metals at Very Low Temperature etc. IV. The Resistance of Pure Mercury at Helium Temperature. In Through Measurement to Knowledge; Springer: Dordrecht, the Netherlands, 1911; Volume 124.

10. Bardeen, J.; Cooper, L.N.; Schrieffer, J.R. Theory of Superconductivity. Phys. Rev. 1957, 108, 1175-1204. [CrossRef]

11. Dickson, P. Sputnik: The Shock of the Century; Walker Publishing Company, Inc.: New York, NY, USA, 2001.

12. See for Example the Historical Development of the US Federal Spending on Defense and Nondefense Research and Development at the Web-Page. Available online: https://www.aaas.org/programs/r-d-budgetand-policy/historical-trends-federal-rd (accessed on 20 February 2020).

13. Marx, W.; Cardona, M.; Lockwood, D.J. Rutherford's impact on science over the last 110 years: A bibliometric analysis Physics in Canada. Phys. Can. 2011, 67, 35-40.

14. Marx, W.; Hoffmann, D. Bibliometric analysis of fifty years of physica status solidi. Phys. Status Solidi 2011, B248, 2762-2771. [CrossRef]

15. Müller, K.A. Paramagnetische Resonanz von Fe-3+ in SrTiO-3 Einkristallen. Helv. Phys. Acta 1958, 31, $173-433$.

16. Müller, K.A. Electron Paramagnetic Resonance of Manganese IV in SrTiO3. Phys. Rev. Lett. 1959, 2, 341-343. [CrossRef]

17. Müller, K.A. Resonance paramagnetic du Cr3+ dans des monocristaux de SrTiO3. Publ. 7ème Colloq. Ampere Arch. Sci. 1958, 11, 150.

18. The Archives des Sciences éditées par la Société de Physique et d'Histoire Naturelle de Genève can be Accessed via. Available online: https://www.unige.ch/sphn/Publications/ArchivesSciences/ (accessed on 1 March 2020).

19. Shengelaya, A.; Zhao, G.; Keller, H.; Müller, K.A. EPR Evidence of Jahn-Teller Polaron Formation in La1-xCaxMnO3+y. Phys. Rev. Lett. 1996, 77, 5296-5299. [CrossRef] [PubMed] 
20. Cardona, M.; Marx, W. Vitaly 1. Ginzburg - A bibliometric study. J. Superconduct. Nov. Magn. 2006, 19, 459-466. [CrossRef]

21. Müller, K.A.; Burkhard, H. SrTiO3: An intrinsic quantum paraelectric below 4 K. Phys. Rev. B 1979, 19, 3593-3602. [CrossRef]

22. Waltman, L.; van Eck, N.J.; Noyons, E.C.M. A unified approach to mapping and clustering of bibliometric networks. J. Informetr. 2010, 4, 629-635. [CrossRef]

(c) 